\title{
Comunicação
}

[Communication]

\section{Efeitos da acupuntura nos acupontos Shenmen (C-7) e Neiguan (PC-6) sobre a frequência cardíaca, a variabilidade da frequência cardíaca e o ritmo cardíaco em cães saudáveis}

\author{
[Effects of acupuncture at Shenmen (C-7) and Neiguan (PC-6) acupoints on the heart hate, hart rate \\ variability and cardiac rhythm in healthy dogs] \\ E.G.M. Silva ${ }^{1}$, L.M.C. Conti ${ }^{2}$, T. Champion ${ }^{3}$, V.R.C. Souza ${ }^{1}$, C.H.T. Mathias ${ }^{2}$, M.A. Lázaro ${ }^{2}$, \\ V.R. Fortunato ${ }^{2}$, F.S. Ferreira ${ }^{4}$ \\ ${ }^{1}$ Médico veterinário autônomo - Vitória, ES \\ ${ }^{2}$ Universidade Vila Velha - Vila Velha, ES \\ ${ }^{3}$ Universidade Federal da Fronteira Sul - Realeza, PR \\ ${ }^{4}$ Instituto Qualittas de Pós-Graduação - Campinas, SP
}

A acupuntura é uma técnica oriunda da medicina tradicional chinesa que visa ao restabelecimento de estados funcionais alterados até o reequilíbrio da homeostase, pela influência sobre determinados processos fisiológicos (Kimura e Hara, 2008). Atualmente representa um dos mais populares métodos de tratamento alternativo e complementar em medicina veterinária, devido a sua longa história e ao aumento das evidências científicas sobre sua eficácia (Xie e Wedemeyer, 2012).

O mecanismo de ação dessa técnica envolve a estimulação de pontos determinados na pele, por meio da inserção de agulhas. Esses pontos específicos são chamados acupontos (Kidd, 2012).

A estimulação desses acupontos pode promover diversas ações. A medicina veterinária tem se utilizado destes para diversos fins, como efeitos neurológicos, ativação do sistema imune próprio, efeitos endócrinos, estimulação da produção de leucócitos, estimulação de nervos periféricos e vegetativos, secreção de serotonina e trombocininas, indução de inflamação asséptica com liberação de substâncias vasoativas, melhorando a circulação local e o controle da dor (Kidd, 2012). De um ponto de vista neurofisiológico, os efeitos de acupuntura são, pelo menos em parte, mediados por uma cascata de endorfinas e monoaminas. As

Recebido em 31 de julho de 2015

Aceito em 24 de novembro de 2015

*Autor para correspondência (corresponding author)

E-mail: felipp.sf@gmail.com contraindicações da acupuntura englobam alterações severas e irreversíveis, como necroses, fibroses, septicemias, distrofias severas, doenças infecciosas com decorrer epidêmico, tumores e indicações cirúrgicas (Draehmpaehl e Zohmann, 1994).

No corpo humano, existem cerca de 365 acupontos. Um dos mais importantes para o controle e a prevenção dos distúrbios cardiovasculares é o acuponto pericárdio 6 (PC-6 - Neiguan), responsável pelo controle sanguíneo, pelas vias de condução e pelos impulsos elétricos (HO et al., 1999). O efeito benéfico da estimulação desse ponto foi demonstrado por angiografias de radionuclídeos de equilíbrio seriadas (HO et al., 1999). Outro ponto fortemente correlacionado às atividades fisiológicas do coração é o acuponto coração 7 (C-7 - Shenmen) (Cárdenas, 2006).

Segundo Cárdenas (2006), estudos terapêuticos já foram desenvolvidos em pacientes humanos com arritmias cardíacas por meio da estimulação dos acupontos coração 7 (C-7 - Shenmen) e pericárdio 6 (PC-6 - Neiguan), os quais demonstraram efeito terapêutico significativo. Entretanto, em medicina veterinária, observa-se na literatura uma grande carência acerca da utilização da acupuntura como estratégia terapêutica não convencional sobre o sistema cardiovascular, sobretudo estudos avaliando seu efeito sobre a variabilidade da frequência cardíaca de cães. 
O objetivo deste trabalho foi avaliar a influência do estímulo dos acupontos C-7 (Shenmen) e PC6 (Neiguan) sobre as atividades autonômicas simpática e parassimpática dos parâmetros cardiovasculares em cães saudáveis, mediante a avaliação do ritmo cardíaco (RC), da frequência cardíaca (FC) e da variabilidade da frequência cardíaca (VFC).

O presente estudo foi aprovado pelo Comitê de Ética para Uso de Animais da Universidade Vila Velha (UVV), sob o registro $n^{\circ} 282 / 2013$.

Foram utilizados 30 cães adultos, saudáveis, de ambos os sexos (18 fêmeas e 12 machos), sem restrição de raça, com idade média de 4,8 $8 \pm 2,7$ anos e pesando em média $15,6 \pm 6,8 \mathrm{~kg}$. Os critérios de inclusão envolveram animais com histórico médico normal, exame físico e eletrocardiográfico sem evidências de doenças cardíacas congênitas ou adquiridas. Foram realizadas, ainda, avaliações hematológicas, como hemograma e bioquímica sérica (ALT, FA, FA, ureia e creatinina). Foram excluídos da pesquisa animais com sopro cardíaco, doenças concorrentes, lesões externas, hematológicas ou eletrocardiográficas.
Para o ensaio experimental, os animais foram submetidos a duas seções de acupuntura, com intervalo de sete dias entre elas. Para a primeira seção, os animais foram contidos fisicamente em decúbito lateral direito, e as agulhas posicionadas sobre os acupontos (ACs) coração 7 (C-7 Shenmen), localizado entre o processo estiloide lateral e o osso cárpico acessório, exatamente na dobra da articulação úmero-rádio-ulnar, e pericárdio 6 (PC-6 - Neiguan), localizado entre os tendões do músculo flexor carporradial e do músculo flexor digital superficial (Fig. 1A, 1B e 1C), conforme descrito por Draehmpaehl e Zohmann (1997). Já para a segunda seção, os animais foram novamente posicionados em decúbito lateral direito, e as agulhas inseridas em acupontos falsos (AF), localizados no oitavo e no nono espaço intercostal esquerdo (Figura 1D), onde não há interferência de nenhum outro meridiano, conforme descrito por (Draehmpaehl e Zohmann, 1994). Independentemente da seção, não foi realizada estimulação manual das agulhas, e os acupontos permaneceram estimulados por um período de 30 minutos, respeitando-se o período mínimo de 10 a 20 minutos sugerido por Scott (2001) para que o acuponto possa promover um efeito observável.

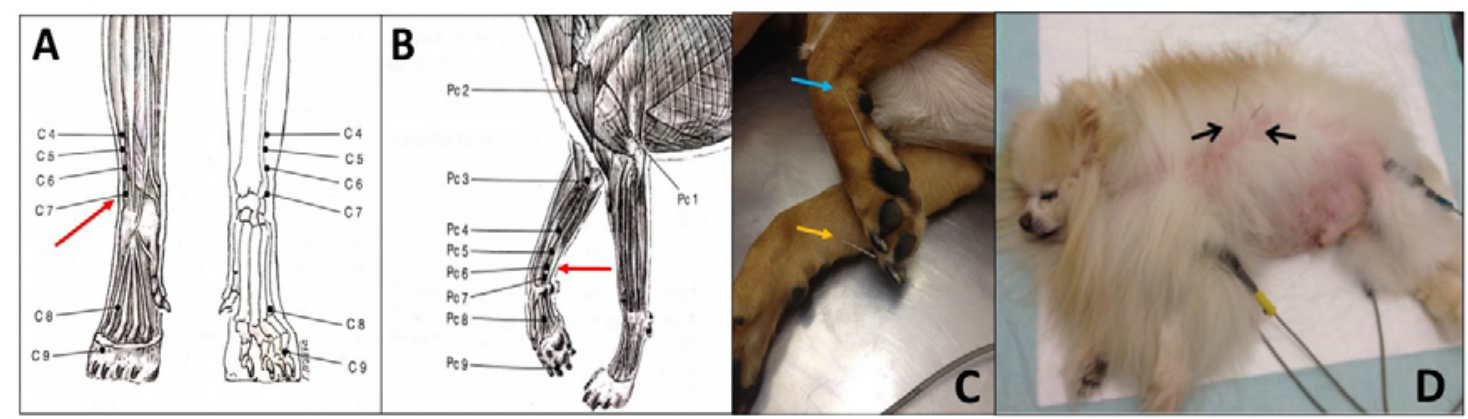

Figura 1. Localização dos acupontos C-7 (seta em A) e PC-6 (seta em B) no cão (Adaptado de Draehmpaehl e Zohmann, 1997). Em C, acupuntura em cão do experimento acuponto C-7 (seta azul) e acuponto PC-6 (seta laranja). Em D, acupuntura em cão do experimento em acuponto falso. Arquivo pessoal, 2014.

Previamente à aplicação das agulhas nos ACs, foi realizado um registro eletrocardiográfico (ECG) com dois minutos de duração e, após a inserção das agulhas nos $\mathrm{ACs}$, o registro eletrocardiográfico se manteve durante os 30 minutos de acupuntura. Para a realização do eletrocardiograma, foi utilizado um aparelho de eletrocardiografia computadorizada (ECGPC $\left.\mathrm{TEB}^{\circledR}\right)$, respeitando-se o padrão de fixação de eletrodos descrito por Tilley (1992), com registro das derivações bipolares e unipolares aumentadas e calibração de $25 \mathrm{~mm} / \mathrm{s}$ e $1 \mathrm{mV}(\mathrm{N})$.

Os dados do ECG foram interpretados de forma comparativa pré e pós-estimulação dos acupontos, sendo os dados pós-estimulação coletados e interpretados em intervalos de cinco minutos até o final da gravação (30 minutos). 
Para a análise da VFC, foi utilizada a fórmula disponível em Carareto et al. (2007). A normalidade dos dados obtidos foi verificada pelo teste de Shapiro-Wilk $(\mathrm{P}>0,05)$, seguida pelo teste ANOVA com pós-teste de Bonferroni para as variáveis paramétricas e pelo teste de Kruskal-Wallis com pós-teste de Dunn $(\mathrm{P}<0,05)$ para as variáveis não paramétricas. Foi realizada análise descritiva para as variáveis qualitativas.

No presente estudo, $90 \%$ dos animais apresentaram arritmia sinusal respiratória, demonstrando maior influência do sistema nervoso simpático, em todos os momentos do grupo ACs, enquanto no grupo AF esse ritmo cardíaco esteve presente em $100 \%$ dos animais, em todos os momentos. O ritmo sinusal foi encontrado apenas em 10\% dos animais do grupo ACs, sugestivamente por se tratar da primeira exposição dos animais ao experimento, resultando em maior influência parassimpática sobre o sistema cardiovascular. Conforme demonstrado na Tab. 1, houve pequena $\mathrm{e}$ irrelevante diferença quando comparada a FC de todos os momentos do grupo ACs com os momentos do grupo AF, o que evidencia, portanto, a ineficácia da estimulação dos acupontos sobre esse parâmetro.

Tabela 1. Frequência cardíaca (FC) e variabilidade da frequência cardíaca (VFC) obtidas com a realização dos acupontos verdadeiros (AV) C-7 e PC-6 e do acuponto falso (AF) em diferentes momentos do ECG, expressos em mediana (percentil $25 \%$ - percentil $75 \%$ ) para a FC e média \pm desvio-padrão para a VFC

\begin{tabular}{ccccc}
\hline Momentos & \multicolumn{2}{c}{$\mathrm{FC}(\mathrm{bpm})$} & \multicolumn{2}{c}{$\mathrm{VFC}$} \\
\hline 0 minuto & $107(92-130) \mathrm{AV}$ & $112(93-129) \mathrm{a}$ & $12,79 \pm 0,46 \mathrm{ab}$ & $12,71 \pm 0,47 \mathrm{~b}$ \\
5 minutos & $90(79-121) \mathrm{a}$ & $93(82-115) \mathrm{a}$ & $13,08 \pm 0,48 \mathrm{ab}$ & $13,09 \pm 0,49 \mathrm{ab}$ \\
10 minutos & $94(79-118) \mathrm{a}$ & $88(70-123) \mathrm{a}$ & $13,01 \pm 0,48 \mathrm{ab}$ & $13,12 \pm 0,53 \mathrm{ab}$ \\
15 minutos & $96(76-120) \mathrm{a}$ & $99(81-115) \mathrm{a}$ & $13,04 \pm 0,52 \mathrm{ab}$ & $13,17 \pm 0,51 \mathrm{a}$ \\
20 minutos & $95(79-120) \mathrm{a}$ & $95(81-112) \mathrm{a}$ & $13,13 \pm 0,51 \mathrm{ab}$ & $13,07 \pm 0,54 \mathrm{ab}$ \\
25 minutos & $104(83-115) \mathrm{a}$ & $89(76-108) \mathrm{a}$ & $13,15 \pm 0,45 \mathrm{ab}$ & $13,17 \pm 0,53 \mathrm{a}$ \\
30 minutos & $87(82-107) \mathrm{a}$ & $95(81-117) \mathrm{a}$ & $13,18 \pm 0,46 \mathrm{ab}$ & $13,11 \pm 0,56 \mathrm{ab}$ \\
& & $\mathrm{P}=0,0890$ & & $\mathrm{P}=0,0051$ \\
\hline
\end{tabular}

Para a FC, valores seguidos por letras distintas diferem entre si $(\mathrm{P}<0,05)$ ao teste de Dunn.

Para a VFC, valores seguidos por letras distintas diferem entre si $(\mathrm{P}<0,05)$ ao teste de Bonferroni.

Não foi observada diferença na VFC (Tab. 1) entre os grupos do ACs e AF, exceto quando comparado apenas o momento basal com os momentos 15 e 25 minutos do AF, contudo sem relevância clínica para o estudo.

Sabidamente, VFC constitui uma medida simples e não invasiva que avalia os efeitos do sistema nervoso autônomo sobre o coração. Variações nessa variabilidade são normais e esperadas, indicando habilidade do coração em responder a múltiplos estímulos fisiológicos e ambientais. Em síntese, uma alta VFC representa boa adaptação, caracterizando um indivíduo saudável, com mecanismos autonômicos eficientes, enquanto a baixa VFC representa adaptação anormal e insuficiente do SNA (Carareto et al., 2007).

Dentre as opções terapêuticas para o tratamento de anormalidades dos mecanismos autonômicos, a acupuntura vem se destacando em vários estudos em animais, entretanto em nenhum desses estudos se objetivou a avaliação do efeito da acupuntura em indivíduos saudáveis, conforme ocorreu no presente estudo, fator limitante à discussão mais aprofundada.

Observam-se, na literatura, diversos estudos em que foram observados os efeitos da acupuntura sobre pacientes com diferentes distúrbios cardiovasculares. Syuu et al. (2001) relataram efeitos benéficos da estimulação do acuponto PC-6 em cães anestesiados e sob toracotomia. Nesse estudo, os autores descrevem reduções na pressão arterial média, volume diastólico final, frequência cardíaca, débito cardíaco e pressão sistólica final mediante estimulação do acuponto. $\mathrm{O}$ efeito cardiovascular da acupuntura sobre o acuponto PC-6 em cães também foi observado por Jingbi (2004) em modelo de experimental de infarto agudo do miocárdio. Nesse estudo, foi observado que a acupuntura proporcionou redução do segmento ST do eletrocardiograma, além de reduzir as áreas de infarto induzidas pela ligadura coronariana. Entretanto, esses dois 
estudos diferem do presente pelo fato de as agulhas terem sido estimuladas eletricamente (eletroacupuntura), enquanto no presente estudo estas permaneceram em repouso. Cárdenas (2006) observou que a estimulação dos mesmos acupontos empregados no presente estudo em cavalos sob anestesia geral demonstrou efeitos positivos sobre a taquicardia ventricular induzida por infusão contínua de dopamina, nos quais a estimulação dos ACs C-7 e PC-6 promoveu diminuição do tempo da taquicardia ventricular, demonstrando, assim, a eficácia da estimulação desses acupontos em animais. Um estudo controlado em ratos demonstrou que a estimulação dos acupontos PC-5 e PC-6 reduziu significativamente a incidência de taquiarritmias induzidas experimentalmente pós-oclusão e reperfusão da artéria coronária esquerda (Lujan et al., 2007). Em humanos, a revisão de oito estudos utilizando acupuntura para o tratamento de arritmias cardíacas encontrou cerca de 87 a $100 \%$ que foram convertidos ao ritmo sinusal após a estimulação dos acupontos (Xie e Wedemeyer, 2012).

Finalmente, Kimura e Hara (2008) desenvolveram um estudo em que foram estudados os efeitos da eletroacupuntura sobre o ritmo do sistema nervoso autônomo em cães. Dentre outras análises, observaram o coeficiente de variação dos intervalos R-R (CVRR) do eletrocardiograma, o qual avalia o índice de atividade nervosa autônoma, de forma semelhante à VFC, a qual também se utiliza do intervalo R-R como unidade de medida. Seus resultados apontaram que, embora alguns elementos analisados tenham apresentado diferenças estatisticamente significativas após a estimulação, o CVRR e a frequência cardíaca não sofreram alterações dignas de nota, de forma semelhante aos resultados observados no presente estudo, ainda que os estudos de Kimura e Hara (2008) tenham sido realizados sob eletroacupuntura nos acupontos Xuan Shu (GV5) e Bai Hui (GV-20), enquanto o presente estudo não se valeu da estimulação elétrica sobre os acupontos falsos ou verdadeiros (C-7 Shenmen e PC-6 Neiguan).

Limitações a este estudo compreendem o fato de que a escolha dos acupontos explorados baseouse nas indicações terapêuticas descritas por Draehmpaehl e Zohmann (1994), em que o acuponto C-7 é indicado em casos de alterações funcionais do coração, e o acuponto PC-6 quando há dores miocárdicas. Ainda segundo os mesmos autores, essa forma de terapia alternativa objetiva normalizar funções orgânicas alteradas e, como no presente estudo foram utilizados animais hígidos, a acupuntura pode não ter promovido influência sobre a VFC nos cães que participaram do experimento. Limita-se, ainda, este estudo quando da observação das recomendações descritas por Scott (2001), quando ele destaca a necessidade de um ou dois tratamentos semanais durante quatro a seis semanas consecutivas, tempo em que se espera que possa haver a resposta desejada à terapia, fato não realizado no presente estudo.

Embora os resultados apresentados apontem limitações, estes corroboram em parte aqueles descritos pela literatura consultada, suscitando, se possível, a necessidade de mais estudos que investiguem a efetividade da acupuntura sobre a VFC e demais parâmetros cardiovasculares em cães hígidos e posteriormente portadores de disfunções cardiovasculares, objetivando-se a melhor compreensão dos possíveis efeitos benéficos sobre o equilíbrio simpatovagal.

Em conclusão, este estudo sugere que o estímulo dos acupontos C-7 e PC-6 não promove alteração do equilíbrio simpatovagal em cães hígidos, especificamente sobre a variabilidade da frequência cardíaca e a frequência cardíaca de cães saudáveis.

Palavras-chave: equilíbrio simpatovagal, frequência cardíaca, acupuntura

\begin{abstract}
Acupuncture derives from traditional Chinese medicine, which aims to restore homeostasis. The action mechanism of this technique involves stimulation of certain points on the skin by inserting a needle. The aim of this study was to evaluate the influence of stimulation of acupoints C7 and PC6 on the heart rate variability, heart rate and heart rhythm in healthy dogs. 30 male and female adult dogs were used, with no breed restriction. The animals were analyzed at two different times in weekly intervals. Firstly were applied needles in the true acupoints, and on the second time we applied needles in false acupoints.
\end{abstract}


Silva et al.

Previously to the acupuncture an ECG with 2 minutes of recorder was performed, and after an insertion of needles the electrocardiographic recording was maintained during the 30 minutes of acupuncture. Results showed no significant difference between the HR when comparing true and false acupoints ( $p=$ $0.890)$. For heart rate variability a small difference was observed between the groups $(p=0.0051)$, however, when comparing the baseline with 15 and 25 minutes of monitoring in the false acupoint group, no significant results were found when compared with true acupoint. In conclusion, it is suggested that the stimulation of acupoints C-7 and PC-6 in healthy dogs does not promote change in sympathovagal balance, specifically on the heart rate variability, heart rate and heart rhythm on healthy dogs.

Keywords: sympathovagal balance, heart rate, acupuncture

\section{REFERÊNCIAS}

CARARETO, R.; SOUSA, M.G.; ZACHEU, J.C. et al. Variabilidade da frequência cardíaca em cães anestesiados com infusão contínua de propofol e sufentanil. Arq. Bras. Med. Vet. Zootec., v.59, p.329-332, 2007.

CÁRDENAS, J.J. Estudo comparativo entre a lidocaína e a acupuntura no tratamento da taquicardia ventricular induzida com infusão contínua de dopamina em equinos sob anestesia geral com halotano. 2006. 14f. Tese (Doutorado) - Universidade Estadual Paulista, Faculdade de Medicina Veterinária e Zootecnia, Botucatu, SP.

DRAEHMPAEHL, D.; ZOHMANN, A. Acupuntura no cão e no gato: princípios básicos e prática científica. São Paulo: Roca, 1994. 245p.

HO, F.M.; HUANG, P.J.; LO, H.M. et al. Effect of acupuncture at nei-kuan on left ventricular function in patients with coronary artery disease. Am. J. Chin. Med., v.27, p.149-156, 1999.

JINGBI, M. The effects of acupuncture in treatment of coronary heart diseases. J. Trad. Chin. Med., v.16, p.16-19, 2004.

KIDD, J.R. Alternative medicines for the geriatric veterinary patient. Vet. Clin. Small Anim., v.42, p.809-822, 2012.
KIMURA, Y., HARA, S. The effect of electroacupuncture stimulation on rhythm of autonomic nervous system in dogs. J. Vet. Med. Sci., v.70, p.349, 2008.

LUJAN, H.L.; KRAMER, V.J.; DICARLO, S.E. Electroacupuncture decreases the susceptibility to ventricular tachycardia in conscious rats by reducing cardiac metabolic demand. Am. J. Physiol. Heart Circ. Physiol., v.292, p.25502555, 2007.

SCOTT, S. Developments in veterinary acupuncture. Acupunt. Med., v.19, p.27-31, 2001.

SYUU, Y.; MATSUBARA, H.; KIYOOKA, T. et al. Cardiovascular beneficial effects of electroacupunture at Neiguan (PC-6) acupoint in anesthetized open-chest dog. Jpn. J. Physiol., v.51, p.231-238, 2001.

TILLEY, L.P. Essential of canine and feline electrocardiography. 3.ed. Philadelphia: Lea \& Febiger, 1992. 470p.

XIE, H.; WEDEMEYER, L. The validity of acupuncture in veterinary medicine. Am. J. Trad. Chin. Vet. Med., v.7, p.35-43, 2012. 